\title{
The Pandemic as a Catalyst for Reimagining the Foundations of Location-Based Games
}

\author{
ARPITA BHATTACHARYA, University of California, Irvine, USA \\ TRAVIS W. WINDLEHARTH, University of Washington, Seattle, USA \\ CASSANDRA LEE, University of Washington, Seattle, USA \\ AMULYA PARAMASIVAM, University of Washington, Seattle, USA \\ JULIE A. KIENTZ, University of Washington, Seattle, USA \\ JASON C. YIP, University of Washington, Seattle, USA \\ JIN HA LEE, University of Washington, Seattle, USA
}

\begin{abstract}
Mainstream location-based games (LBGs) highlight three foundations-exercise, exploration, and social interaction-which were hugely disrupted by the COVID-19 pandemic. This genre has already had access issues depending on the player's region, identity, ability, and technology, which the pandemic also amplified. Thus, the pandemic provided a unique opportunity to re-imagine the design of LBGs to preserve player benefits while challenging these foundations. We conducted a two-week diary study with 11 LBG players coping with difficult life circumstances, followed by remote co-design sessions. Participants continued to play primarily to maintain community connection while adjusting the mode of socialization, finding meaning through virtual exploration, and support mental health and personal growth. Participants discussed ways to enhance the experience by expanding the range of physical activities during social distancing, reimagining proximity-based social interactions, and exploring via social construction of places while expanding beyond how the three foundations are currently supported in LBGs.
\end{abstract}

CCS Concepts: • Human-centered computing Human computer interaction (HCI) Empirical studies in $\mathrm{HCI}$

KEYWORDS: Location-based games, Pokémon GO, Ingress, Harry Potter: Wizards Unite, game design, places, wellbeing

ACM Reference format:

Arpita Bhattacharya, Travis W. Windleharth, Cassandra Lee, Amulya Paramasivam, Julie A Kientz, Jason C. Yip, and Jin Ha Lee. 2021. The Pandemic as a Catalyst for Reimagining the Foundations of Location-Based Games. In Proceedings of the ACM on Human-Computer Interaction, Vol. 5, CHI PLAY, Article 280 (September 2021), 25 pages, https://doi.org/10.1145/3474707

\section{INTRODUCTION}

Digital games have played a prominent role in the lives of many people $[16,48]$, where the experience has an overall impact on people's behaviors and wellbeing [23]. As such, researchers

Author's addresses: A. Bhattacharya, University of California, Irvine, Irvine, California 92697 USA; T.W. Windleharth, C. Lee, A. Paramasivam, J.C. Yip; J.A. Kientz, and J.H. Lee, University of Washington, Seattle, WA 98195 USA.

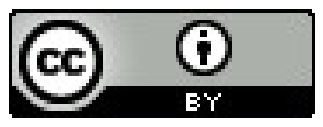

This work is licensed under a Creative Commons Attribution International 4.0 License.

(C) 2021 Copyright held by the owner/author(s).

2573-0142/2021/9-ART280. https://doi.org/10.1145/3474707

PACM on Human-Computer Interaction, Vol. 5, No. CHI PLAY, Article 280, Publication date: September 2021. 
have been examining the influence that digital games have on wellbeing and physical and mental health [24]. One type of game that has received much recent attention is Location-Based Games (LBG) on mobile devices, such as Ingress, Pokémon GO, and Harry Potter: Wizards Unite (HPWU). This genre of video game employs gameplay that progresses based on a player's physical location. LBGs depend on mapping technologies (e.g., GPS) to allow players to indicate their physical location. Various mobile devices (e.g., smartphone, tablets) are used as the primary devices in which LBG players achieve their goals. Because mobile LBGs depend on physical mapping and movement in the real world, players typically meet in physical locations to collaborate, communicate, socialize, organize, and play together.

In July 2016, Niantic, Inc. released Pokémon GO (PoGO), the most popular and mainstream LBG [36]. The game was played by 65 million monthly active users in 2018 and held $84 \%$ of the augmented reality games market share by the end of that year and continued growth in revenue in 2020 with an estimated 166 million players [36]. Ingress and HPWU also have smaller but dedicated player communities. Niantic designs its LBGs based on three foundational pillars, where the game encourages "exercise, exploration, and social interaction" [40]. The core affordances of LBG to physical/mental health, exploration, and social interaction are deeply embedded in the literature, even prior to Niantic's articulation of these as their three pillars. Laato et al. (2019) [28] noted LBGs "are shown to promote exercise, encourage social interaction and increase geographical and cartographical knowledge" by studying the larger body of LBG via software search and review. Avouris et al. [2] study an earlier body of ARGs and exploration, social interaction, and physical activity were all common themes.

In March 2020, four years after the release of PoGO, the COVID-19 pandemic commenced, which drastically changed the ways people interacted in-real-life. With quarantines, restricted travel, the closing of schools, offices, and public spaces, and limited physical interactions at 3 meters (6 feet) of separation, people's social engagement shifted. During the COVID-19 pandemic, we observed rapid changes severely limiting two of the main objectives that defined LBGs - faceto-face social interactions and the ability to freely explore the real world. In addition, there was growing evidence that people pre-COVID-19 depended on and used these games for various health benefits (e.g., [25]). However, several areas were in lockdown during the time when this study was conducted. While limited interactions were possible, the social interactions our participants craved included not only remote co-play but IRL co-play (such as raids in PoGO) and community interactions. LBG developers started making substantial changes to the gameplay, reporting that the pandemic changed how their users play these games [37]. By the end of 2020, developers and management from Niantic shared the difficulty with addressing rapidly changing pandemic situations stating that "the current issue [of COVID] is existential to our game"[38]. Even pre-COVID-19, LBGs presented access issues based on the player's region, identity, ability, and mobile device access, which the pandemic also amplified.

Laato et al. studied how the LBG developers were adapting the design to support players while following government guidelines of social distancing [28]. They found tensions in online LBG player communities in attributing responsibility for social distancing to players versus the game developers in continuing engagement from home as the core intentions of the design features for encouraging outdoor activity and socialization were challenged [27]. Niantic introduced some remote features to alleviate the physical gameplay dependent on movement such as remote passes 
for raiding in $P o G O$, remotely battling with others in Fortresses called the Knight bus in HPWU, and remotely hacking portals using drones (or Dronenet) in Ingress [9,27]. In surveys among LBG players across different lockdown regions, participants self-reported reduction in game play time for LBGs, increase in non-LBG video game playing time, decrease in social behavior, physical activity, and poorer mental health compared to before the pandemic [9,26]. At the same time, ongoing LBG adaptations continued to provide players with avenues for isolated outdoor physical activities, virtual socializing, and providing relief from the stress of the pandemic and difficult news $[9,27]$. The pandemic provided us with an opportunity to examine and re-imagine LBGs as these foundational pillars were disrupted, which radically impacted the players' physical behavior.

Given that LBGs were designed based on foundations that may no longer be possible during a pandemic, what kinds of game designs can we imagine that allow for meaningful gameplay despite the constraints imposed by COVID-19? For instance, what other kinds of socialization can happen without raiding together [4]? Are there additionally supported health benefits other than walking? Can people discover locations without being able to go there physically? In this paper, we discuss how the current foundations for designing and implementing LBGs influenced gameplay during the pandemic and contribute to the design changes that could advance the conceptualization of LBGs. In this research, we address the following research questions:

$\boldsymbol{R Q 1}$. What kinds of adjustments are players of LBG and their communities making during the COVID-19 pandemic as key pillars of this genre (i.e., exploration, community, and health) are disrupted?

$\boldsymbol{R} Q 2$. Based on their experience before and during the COVID-19 pandemic, how do players reimagine the design of LBGs to improve support for exploration, community, and health?

To answer these research questions, we conducted a 7-day diary study with 11 participants followed by remote synchronous and asynchronous co-design sessions with 10 of those participants. We present three key themes on how social distancing and lockdowns impacted the three foundations of outdoor physical exercise, real-world socializing, and exploration, subsequently influencing the players' gameplay. We found that although gameplay was altered, players continued using LBGs to (1) preserve and discover historical, social, and personal meaning in virtual and physical locations in the game, (2) preserve and increase virtual and/or proximitybased interactions with their gaming communities; and (3) support personal mental wellbeing and growth. We build on the design ideas of participants and prior work to further the discussion of how we can envision the future of the LBG genre. We engaged with players who used LBGs for health management in co-design to understand the empirical needs and design needs for LBGs that should be considered by future designers and researchers working in this space. We reflect on and identify design recommendations to holistically leverage the design space of interactions between real-world places, people, and augmented reality to support meaningful experiences for players. Informed by prior work, we speculate how the recommendations may be implemented in future LBGs. 


\section{RELATED WORK}

\subsection{Location-Based Games}

Augmented reality games (ARGs) use "technology to superimpose information, sounds, images, and text, on the world we see" (p.1 [22]) and employs the ability of mixed reality games to digitally project graphics and content on the real-world using platforms such as mobile phones. Locationbased games (LBGs) use technology to link physical locations in the real world to game systems, typically requiring players to be in specific places at specific times to accomplish game goals. Some modern games have adapted affordances of mobile devices (e.g., GPS, cameras) to create new LBGs and ARGs for ludic (leisure) and/or pedagogical purposes supporting gaming formats such as action, role playing, and treasure hunt [2].

In mainstream gaming, Niantic, the developer of Ingress, Pokémon GO, and Harry Potter: Wizards Unite (HPWU), serves the highest number of daily active users with these three games, which they explicitly designed upon three pillars; their mission is to "use emerging technology to enrich the experience of the physical world while encouraging exercise, exploration, and social interaction on a global scale" [40]. The mechanics of these games incentivize exercise and exploration by requiring movement from point-to-point to accomplish goals such as "hacking portals" in Ingress to collect in-game gear, catching a digital creature in PoGO, and obtaining special rewards based on distance moved (Appendix A). Notable sites in the world such as sculptures, historical markers, and landmarks become important nodes in gameplay (such as portals in Ingress, or PokéStop in PoGO). Players must visit these sites to collect resources in game and use them to accomplish game goals. The final design pillar, social interaction, is facilitated through cooperation and competition in gameplay. Each of these games have tasks that require multiple people to accomplish, facilitating interaction and coordination between players, often leading to robust communities of practice [64].

After the success of PoGO in 2016 (45 million players in 2016) [57], many of the games that followed were very similar, requiring players to travel around the world, visit augmented sites to gather resources, and use these resources to collect things or fight. Examples include furassic World Alive (2017), Ghostbusters World (2018), and Minecraft Earth (2019). None of these games have come close to the popularity of PoGO. The challenges of designing, launching, and sustaining successful LBGs are exacerbated during the COVID-19 pandemic, and games like Minecraft: Earth had to be shut down due to low engagement [18].

\subsection{Communities and Location-Based Games}

Multiplayer games facilitate or directly require social interaction, and communities often organize around games with active player bases. Social interaction is an important appeal of video games, as players seek to spend time with family and friends, and seek connections and fellowship $[20,54]$. Building on the social appeal of video games, there is a growing body of work describing the development and roles of several types of video game communities, such as communities to facilitate information sharing and collaboration to solve puzzles in ARGs [39], participation of players in online affinity spaces [46], and the development of communities of practice around specific games [44]. 
Video game players often congregate in "affinity spaces", where people come together and learning happens [10]. These social spaces, typically online for games, connect people with information and resources [46]. The construct of "communities of practice" (CoP) [31] describe groups of people connected by a shared domain of interest (in this case, a specific game), and the manners in which they create, learn, develop, and enforce norms, and draw from and contribute to a shared body of knowledge that resides in the community. Video games with more complex mechanics and social roles, such as massively multiplayer online games, ARGs, and LBGs are often the basis for a community of practice. Lau and Agre describe the formation and practices of a World of Warcraft (WoW) CoP [30]. Bogost notes "the people who play video games develop values, strategies, and approaches to the practice of play itself" (p. 119 [6]). Communities of practice have also formed around LBGs, to address the unique challenges of this game genre. For Ingress $\mathrm{CoP}$, researchers found that the community had developed detailed practices around information security, safety, and identity protection, which are unique considerations for this kind of game [64].

\subsection{Exploration in AR games}

The design aspects of LBGs play into a series of constructs about exploration and society, and the design of cities and common spaces, such as smart or playable cities, and facilitate "placemaking". Smart Cities refer to "the introduction of digital technology by governance bodies and its use by service providers and other economic stakeholders to allow a more efficient use of resources, transportation infrastructure, and an increase in citizen safety" [43]. In addition to technology and governance, Chourabi et al. identify six other critical factors of smart city initiatives, including physical and natural spaces, and people and communities, which they believe have been neglected in favor of technology and policy [7]. Wolff et al. also make the case that games are an effective mechanism for connecting these "smart citizens to smart cities" (p.164, [65]). Schouten et al. describe games and game mechanics as effective means to better understand complex problems such as community building and participation [52]. Learning about urban areas can also be facilitated through LBGs both using system or designer generated narratives (e.g., [41]) and supporting players in socially constructing their own narratives and riddles using authoring tools [63]. For example, Sintoris et al. used a LBG version of the social game Mafia to facilitate learning about urban areas [55].

LBGs have also been connected to the concept of "playable cities", which refers to the intentional use of designed spaces to facilitate play in contested social spaces, in order to strengthen individual connections to locations and communities [43]. LBGs serve these same functions in social spaces, by augmenting spaces with information and game mechanics [43]. These same mechanisms also help facilitate a process of "placemaking". Innocent explains that placemaking occurs while playing LBGs, which helps establish and reinforce a "place identity" [19]. The idea of space and place [8] is not new and has been conceptualized in early work on LBGs. For instance, Nicklas et al. [41] explain the basic properties and classification of LBGs, discussing "location aware games" where geographical positions of players matter and "spatially aware games" which integrate the real world surroundings of the players. 


\subsection{Video Games and Wellbeing}

Four domains of positive benefits in video games are social, cognitive, motivational, and emotional [12]. Video games can train healthy adult populations on cognitive and emotional skills [45]. Halbrook et al. argue for the notion of an optimal gaming profile for players to support positive living and social activity [14]. Video games with features to support social activities can be beneficial for psychological wellbeing and prosocial behaviors. HCI researchers are also examining how video games can be used for addressing, assessing, promoting, and treating mental health for players, stakeholders, and caregivers [5]. Recent reviews also show that playing video games can positively enhance wellbeing, problem solving skills, social relationships, and physical activity $[1,23]$. Exergaming can be a novel method to increase and/or substitute for short term physical activity $[60,66]$.

Despite positive factors, players that engage in excessive addictive behaviors or focus solely on competitive, achievement-only orientations tend to not gain the positive benefits [14,33]. Players who perceive their lives as satisfying are often more likely to play games in moderation with positive psychological wellbeing [49]. Real-world need satisfaction and frustration often predicted wellbeing better than video game need satisfaction [59]. In contrast, high amounts of compulsive video gameplay are more related to real-life psychological needs. Addictive levels of video gaming are associated with poorer health status and more behavior problems [37] and video game addicts tend to have increased emotional difficulties (i.e., depression, anxiety) [42]. Competitive gaming is associated with decrease in prosocial behavior, but mostly in children (ages 7 - 11) that played video games in excess [33]. Finally, systematic reviews of video games show that representation of women in video games can lead to female self-objectification and negative body images in women [11].

Overall, the research around video games and wellbeing is not about what is "good" or "bad", but rather, what is the effect of wellbeing on players as it pertains to the contextual factors, motivation behind gameplay, and moderation. We focus on how video games help us understand what life experiences affect players, and how and why people play video games, specifically to manage health and wellbeing. Our work examines how players used LBGs to cope during COVID19 and how they envision future LBGs, particularly when fundamental aspects of the game can no longer be accessed due to physical and social limitations.

\section{METHODS}

To understand adjustments that players of LBG and their communities are making during the COVID-19 pandemic (RQ1), in February 2020 (onset of COVID-19), we invited players who use LBGs for coping to fill out a screening survey about their perceived benefits and harms of LBGs and the severity of health conditions they managed using LBGs (if any). We posted the screening survey on social media groups for PoGO, Ingress, and HPWU, inviting players to participate in a 7day diary study and a follow-up co-design session to reimagine the design of LBGs to improve support for exploration, community, and health (RQ2). Each participant was compensated with a \$25 Amazon gift card on completing the entire study. This study was determined to be exempt by our University's Human Subjects Division. 


\subsection{Participants}

Before widespread shelter-in-place orders were made in North America, we received 94 responses to our screening survey and selected 15 participants based on the type and severity of health challenges they manage (3-5 severity rating on a scale of 1 being not-at-all severe and 5 being extremely severe) and their perceived role of LBGs in their wellbeing. After the shelter-in-place order came into effect, we reached out to selected participants asking if they still played LBGs during the pandemic. Twelve participants consented to participate, 11 (labelled P1-P11, Table 1) completed the diary study, and 10 completed the co-design sessions. Six of these participants completed the co-design session synchronously on Zoom video chat in May and June 2020, respectively. Due to difficulties in scheduling synchronous meetings and differences in time zones, it was not possible to schedule the remaining participants for a synchronous video chat (Zoom) session. For accessible participation, we then invited all 11 participants to join an online server on Discord $^{1}$ which we facilitated using the method of Asynchronous Remote Communities [3,35]. Seven participants (including three from the Zoom co-design sessions) completed the co-design activities on Discord in July 2020 (study protocol: Appendix B).

Table 1: Demographic information of participants (end survey was answered by 9 participants, the remaining participants are included as "no response")

\begin{tabular}{|c|c|}
\hline Gender & Female $(n=4)$, Male $(n=6)$, Non-binary $(n=1)$ \\
\hline Age & $20-51$ years $($ mean $=34$ years $)$ \\
\hline Race & $\begin{array}{l}\text { White }(n=4) \text {, Asian }(n=2) \text {, Native American or American Indian } \\
(n=2) \text {, Other }(n=1) \text {, No response }(n=2)\end{array}$ \\
\hline Ethnicity & Hispanic/Latino/Spanish Origin $(\mathrm{n}=3)$ \\
\hline Type of area & Urban $(n=4)$, Rural $(n=3)$, Suburban $(n=2)$, No response $(n=2)$ \\
\hline Household income & $\begin{array}{l}\$ 100 \mathrm{k}-\$ 150 \mathrm{k}(\mathrm{n}=1), \$ 50 \mathrm{k}-\$ 75 \mathrm{k}(\mathrm{n}=1), \$ 35 \mathrm{k}-\$ 50 \mathrm{k}(\mathrm{n}=3), \$ 20 \mathrm{k}-\$ 35 \mathrm{k} \\
(\mathrm{n}=2) \text {, Less than 20k (1), No response (3) }\end{array}$ \\
\hline Number of years playing LBGs & $1-2$ years $(n=1), 4-5$ years $(n=4), 6-7$ years $(n=4)$, No response $(n=2)$ \\
\hline Location-based games played & $H P W U(\mathrm{n}=5)$, PoGO $(\mathrm{n}=7)$, Ingress $(\mathrm{n}=8)$ \\
\hline $\begin{array}{l}\text { Amount of time LBGs games } \\
\text { were played before shelter-in- } \\
\text { place orders (March 2020) }\end{array}$ & 3-30 hours/week (mean =14 hours/week) \\
\hline $\begin{array}{l}\text { Health conditions that players } \\
\text { are navigating }\end{array}$ & $\begin{array}{l}\text { Heart and respiratory disease, Chronic pain, Cancer, ADHD, Social } \\
\text { anxiety, Severe depression, Chronic illness (undisclosed) }\end{array}$ \\
\hline
\end{tabular}

\footnotetext{
${ }^{1}$ Discord: https://discord.com
} 


\subsection{Study Procedures}

Adapting to remote research during the pandemic, we conducted a 7-day diary study followed by two online co-design sessions on Zoom and a 1-month asynchronous co-design study on Discord.

Diary Study: We conducted a diary study with 11 participants (labelled P1-P11), which was completed in March 2020. We gave participants a period of two weeks to log seven days of diary entries on the days they played one or more LBGs. Preliminary analysis of the diary study data informed the protocol for co-design sessions on Zoom and Discord. As the context of play rapidly changed during COVID-19, we also included questions specific to playing LBGs in limitations due to the pandemic.

Design Sessions: We then conducted two online synchronous co-design sessions on Zoom for 120 minutes each with three participants and three researchers (total six members in each session). One researcher facilitated the session, and the others took notes and prompted questions. Researchers collectively had experience playing all the LBGs and participated by sharing their involvement with the gaming community. We used the first 15-20 minutes for video chat testing and trying out an online collaborative whiteboard tool, MURAL. In the next 30-45 minutes, we facilitated focus group discussions on the reasons that participants started and continued playing LBGs during the pandemic. We prompted participants to discuss how the pandemic changed their gameplay and coping strategies. In the last hour, we asked participants to brainstorm and design how they might envision LBGs that provide similar social benefits, health benefits, and opportunities for new exploration, or perhaps different kinds of benefits that would be appealing to them. We asked participants to share as many ideas as they could using digital sticky notes on $\mathrm{MURAL}^{2}$ for 20 minutes (Appendix E). Researchers then grouped these ideas into themes and asked participants to vote on one theme to elaborate on.

Asynchronous Discord Session: We created a server on the chat protocol Discord with four separate channels. Seven participants joined the server between June 30th to July 30th, 2020. All participants were already familiar with Discord for gaming. The discussion prompts in the \#intro_and_gaming_experience and \#gaming_in_pandemic channels were facilitated in parallel whereas the discussion prompt in the \#design_ideas channel was staggered by a week. We waited for all participants to post in a channel before moving onto the design activity. A fifth channel called \#wish_list_for_LBG was created upon participants' request to share their current grievances and wish list related to PoGO, Ingress, and HPWU.

\subsection{Data Analysis}

The co-design sessions on Zoom were transcribed and chat data from Discord was pasted into a document for analysis. Researchers read and discussed the transcripts, session notes, and diary data to conduct affinity modeling (Appendix $\mathrm{C}$ ) and preliminary inductive analysis while writing memos. We then prepared a codebook (Appendix D). Three researchers coded the transcripts, Discord data, and diary data based on this codebook, iterated on the codes, wrote memos, and

\footnotetext{
${ }^{2}$ Mural: https://www.mural.co/
} 
discussed any discrepancies. We identified key themes from the analysis and combined them into memos. The final themes presented in this paper emerged from iterations through discussions and writing with all researchers. We focused our recruitment on people who were managing difficult health challenges and continued to use the game for their health management that participants self-identified in a positive way. Our work aimed to include these participants' lived experiences and expertise in gaming and long-term health management through the co-design process which can be also beneficial to other players in sustainable use of the game for their wellbeing.

\section{FINDINGS}

Three key themes emerged from our analysis of participants' experiences playing LBGs while adapting to changing circumstances and expectations during the COVID-19 pandemic (RQ1): (1) ascribing meaning to exploration and player representation, (2) adapting to physical limitations and social distancing, and (3) mental wellbeing and community oriented real-world action.

\subsection{Ascribing Meaning to Exploration and Player Representation}

Before the COVID-19 pandemic, participants enjoyed traveling to play LBGs and learning about the history and interesting structures of certain areas associated with portals, PokéStops, gyms, and inns. Locations had specific meanings for the participants such as learning about the place they lived in, interesting historical sites, and cities they visited with a significant other that made the experience memorable and/or enjoyable. For some, exploration was not intentional but more of an enjoyment of discovering something unexpected. P9 would look up more information on where her $H P W U$ and $P o G O$ gifts are from which helped her learn about the site.

What I have loved so much about Ingress is that it has taken me to see historical sites in my own community, in my own area that I never knew were there - and because I love history. With the Harry Potter and the Pokémon Go gifts that people send, I would always look up the name and then go and try to find it on the portal map what I find out about. Like 'this is so cool', 'that this person sent me this' and I want to learn more about it. - P9

During the co-design sessions, participants wanted to include more meaningful aspects of culture and special interests in the gameplay. Missions ${ }^{3}$ and Tessellations ${ }^{4}$ (a global media hunt) which require community members to organize in Ingress encouraged players to customize gameplay in specific communities and learn about cultural and historical facts.

We are playing this thing called the Tessellation, which is a global game that requires you to connect with other people in regards to history, local knowledge and of course, some Ingress related lore. It's good fun. You get two to three hours of guessing around, getting to know more about culture and historical facts that you probably never would have known of. - P7

\footnotetext{
${ }^{3}$ Missions: https://fevgames.net/ingress/ingress-guide/ops/missions/

${ }^{4}$ Tessellation: https://community.ingress.com/en/discussion/10527/tessellation-medals-faq
} 
However, players found that the current Niantic portal approval process for creating customized community informed portals was difficult and ambiguous. P9 explained that the approval of new portals was difficult without good feedback to the players on improving rejected applications. Interviewees shared that for strategic reasons, sometimes players would refuse to approve certain portals in Ingress because it potentially benefited the other team (e.g., area known for dominance of a certain team, potential couch/desk portal for active players).

P6 was passionate about her culture and representation through Ingress. She enjoyed being able to travel to different cities and represent herself and her community through custom-designed clothing and accessories, which she also distributed among other players in her faction. Participants, however, spoke about lacking diverse cultural representation in mainstream media and safety issues due to skin color and stigmatized perceptions in areas where they are a racial minority. During the COVID-19 pandemic, this resulted in them feeling excluded during the GO Fest live stream where the majority represented were White.

The livestream contained many pictures of White women, and I didn't see any Indigenous or Black trainers featured at all. As a Native trainer, I have been profiled by security, I have been targeted as suspicious while playing in well-lit areas while a White man ten feet away wearing all black is seen as "normal". The livestream would have been a great time to amplify voices of Indigenous or Black trainers, but Niantic seems to just ignore us. I love playing Ingress on [anonymized]. But my own personal rule is always go with at least one White person. It's a lot safer for us BIPOC that way. Same thing with places like [anonymized]. - P6

Representation matters. It's been an ongoing issue for my all of my friends that are PoC. A few really really loved the idea of Ingress but didn't feel safe playing it because not being arrested/shot/killed was a higher priority than a game. - P1

\subsection{Adapting to Physical Limitations and Social Distancing}

Due to the need for physical and social distancing during the pandemic, players adapted their gameplay to leverage remote features introduced by Niantic and expressed the need for additional alternative gameplay. P9 mentioned trying out drones (Dronenet) that allow players to hack portals remotely within its visual range in Ingress. It took her longer to achieve her goal due to the wait times because the drone should remain at a portal for at least 60 minutes before it can be moved to another portal. Mobility restrictions and changes in routine led to reduced gameplay during the pandemic. P8, who primarily played while going for walks in his neighborhood, explained that he walked much less than before the pandemic.

Obviously, I don't play as much because of the current situation. Whenever I go out for walks in my neighborhood, I always have Pokémon GO out. It's just something to do while walking around to make it more entertaining and it's a way to explore the world. - P8

Limitations in the ability to play LBGs before the pandemic included physical access issues, safety, lack of resources tied to physical locations in rural areas, monetary needs, and weather 
issues. P1 recalled encountering these equity issues during her cancer treatment which were exacerbated during the pandemic for majority.

It's way harder to level in Ingress when you are stuck in a hospital and banned from social interactions most of the time - Having phone-based games to play and the resulting community from them has actually helped [me] a lot in the sanity department. Stem cell transplants are pretty brutal. I will say that hospitals need more portals though - I live right at the edge of rural [area] and unfortunately pretty physically limited from all the surgeries and chemo. $-P 1$

The second pillar of real-world social interactions in LBGs was heavily impacted for many of our participants as they strongly identified with and felt connection to their gaming community. P7, P9, or their family members were in vulnerable groups, so they took additional precautions. Some participants used portals at their home location and played in their cars (called "car-gressing" by Ingress players but also used in the context of HPWU and PoGO). This defeated the purpose of being physically active but facilitated social connection as they actively shared resources through these portals.

I do have a 'couch portal' [referring to a portal at the player's house in Ingress]. So, I've been farming, a lot of gear. People swing by my apartment and I drop off gear so that they can go out and play. Our normal farm locations are way too crowded right now. - P4

We have had a couple fields [in Ingress] where everybody just drives up in their car. We wave at each other out the window and drop resonators [game items], and we don't get out of our cars to do anything. So that's how I've been car-gressing. - P9

P3, who did not strictly follow social distancing guidelines, expressed guilt around meeting other players.

I do miss getting together with small groups for coffee and trades. It was also nice to chat during raids - We tried last weekend to gather with 3 others in their front yard, but even that broke rules. We just aren't wired that way. $-P 3$

Some events that the players and their community were looking forward to were cancelled, postponed, and/or redesigned to be virtual to prevent crowds from forming. These included larger official events like GO Fest or Safari Zone events in PoGO, First Saturday in Ingress, as well as smaller local gatherings.

Definitely not the same without ppl around to talk to. About the only way we get to "see" each other -now that we have play at home options built into the system it is a little better but I still miss the social face-to-face aspect of it. My local community has done First Saturday for Ingress but with not actually meeting up it doesn't FEEL the same to me and that's what got me (and kept me) playing Ingress. Without the social interactions Ingress just sits on my phone unopened a lot. - P10

Virtual social connections with their LBG community also provided positive benefits and reduced loneliness for all participants in an otherwise isolating time. Similar to results from Ellis et 
al. [9], our participants also shared that these social interactions were a major reason why they continued playing LBGs during the pandemic and it transcended physical limitations and boundaries and enabled players to communicate with people around the world. Some players adapted to play other LBG games and non-LBGs together with their LBG team players (e.g., P1 played Animal Crossing and WoW).

Had an Ingress First Saturday! Got to talk to Ingress agents on videoconferencing from around the world. It was awesome to meet some agents in Taiwan-We both have disabilities, so these events are a great way for us to get some exercise and support each other during the COVID-19 pandemic. - P6

The pandemic brought economic hardships and players explained that LBGs provided an inexpensive way to strengthen current relationships with friends and family.

I'm furloughed. I don't have the budget for a [Nintendo] Switch. I don't have the budget for new fancy equipment. That is one big reason why I have played all of the Niantic's games so consistently, it's because I don't need to go buy a gaming system to play them. - P9

\subsection{Mental Wellbeing and Community amid Difficult Life Circumstances}

LBGs supported mental wellbeing of players during difficult life circumstances including the impact of the pandemic in two key aspects: (1) improving agency, activation, and sense of achievement and (2) providing real-world community support outside gaming.

\subsubsection{Agency, Activation and, Achievement}

Players found LBGs helpful to get themselves active and outside while physically distancing, sustaining the strategies participants were using before the pandemic to cope with their physical and mental health conditions as much as possible. In crisis situations such as the COVID-19 pandemic, where real-world factors were largely out of an individual's control and blurred worklife boundaries, gaming provided a place of distraction, agency, and sense of accomplishment as the players felt activated (e.g., run errands outside while playing the game) [17]. In their diary entries, players attributed improvement in physical and mental health to LBGs motivating them to take appropriate breaks from work, having something to look forward to, and finding time to relax from the stress. P6 also found Ingress helpful in regulating her ADHD.

[I] have ADHD, so it helped me focus by giving something to do while walking. - P6 (Diary)

It sounds pathetic but PoGO gives me something to look forward to every day. I should be cooking \& cleaning but I hate that. I like organizing Marie Kondo style but don't feel motivated for it. PoGO allows me to lose myself in flow, which is supposed to be an indication of enjoyment. Trading on my husband and my phones puts me in a calm rhythm I can't get from meditation because my mind is too busy-It positively impacted my wellbeing by giving me something concrete to focus on, which helped avoid worrying. - P3 
Honestly, it's been very mentally and emotionally draining for me not able to go out into the community and do anything. [I'm a] very goal-oriented person and not having anything to do has been very hard for me. So, with Harry Potter, the daily challenges, the Knight Bus, the feeling like I can communicate with my community. I can be a part of things. I can reach goals [and that] has been incredibly helpful for me in terms of my mental health. $-P 9$

P3 explained how going outside for PoGO helped with their agoraphobia. They also explained tensions in how the game negatively impacted their sleep, but at the same time helped them cope with PTSD.

I was negatively impacted by some stress of preparing for competitions and trying a new remote raid method. I also got less sleep than usual/ideal. However, this exhaustion helped avoid my non-military PTSD nightmares/flashbacks. - P3 (Diary)

\subsubsection{Real World Community Support.}

Playing LBG provided meaningful relationships for players, where they could rely on their community during times of emotional and financial distress in the real world. During the pandemic, P7's community created a space dedicated for allowing people to talk about COVID-19 to vent and share information.

I've seen people come to Ingress due to a [sic] health issues, due to a need for social interactions. Some people found our communities to become sort of like a support group for sometimes very difficult times. I don't know if there would be some way to do that through this. We've even had created a chat, just for Covid-19 discussion. People vent out their frustrations, or just share useful information to avoid fake news which is nice. - P7

In addition, the emotional support from the community extended to material support in real life. P9 who has an autoimmune disease, received real world support from her community through grocery deliveries and in turn had been distributing home-made masks.

Well, obviously the pandemic has changed things greatly. I have an autoimmune disease so I've been leaving the house, maybe once a week. But when everything first started happening, my community was really awesome, people brought me food, people checked in on me. And that was amazing - We have all been asked about masks. I sew. I have made masks for everybody in my area. There's been a bag on my, on my front doorknob just if you need one drop by, pick one up. But we have all been very, very, very cautious about human interaction and wearing masks and bringing your hand sanitizer - not getting out of your cars and just waving. - P9

This was not a one-off event as participants also found support through their gaming community during life transitions such as moving and loss of jobs and recalled instances of organized efforts for collective support in the pre-pandemic times. For example, P7's Ingress community worked together to provide emotional and financial support and she has benefited personally during flooding damages in her area. 
That people that you actually get to connect with care about you as a person. I mean, also as a player, of course, but also as a person and I've seen many instances of this with people that were on cancer treatment or just had a bad accident or just somebody that had their mother, like in the other side of the country and you couldn't personally reach her and then your teammates from the community would go visit her. It's amazing. I remember once we had a very bad flooding in the northern area of my country and people actually sent money to help us up with getting stuff for reconstruction and such. $-P 7$

\section{DESIGN IDEAS FOR REIMAGINING LOCATION BASED GAMES}

In the co-design sessions for reimagining the design of LBGs (RQ2), participants highlighted the importance of preserving and enhancing aspects of LBGs to support players in creating and remembering meaningful experiences through exploration, reimagining proximity-based social connection, and expanding the range of healthy activities (summarized in Table 2).

\subsection{Supporting Meaningful Experiences through Exploration}

Participants did not just want a virtual mobile game but wanted to preserve the gaming experience of exploring the virtual and physical space and social roles in creative and meaningful ways. Players wanted more engagement with artifacts in the game that were culturally and/or socially meaningful to them in addition to reading a description. In co-design session 2, P8 suggested adding special rewards on historical sites in addition to having basic background information about the site such as date of creation. P7 and P9 brainstormed ideas for customizing gameplay and adding conscious efforts and challenges for historical and cultural learning, such as using clues during days when there are special events (e.g., First Saturdays and Mission days).

Maybe [on] Mission days, we could have like a prerequisite for at least one or two missions that actually had a code, a pass phrase related to the history of culture or culture - if you could actually make that our department would make it more cultural, I think. - P7

Players in the Discord group brainstormed increasing the complexity of gameplay by mixing AR/VR formats to utilize the space. These included adding clues, narratives, and using the physical space and augmented reality as a part of narrative by distributing distinct roles and strategies among team members. Participants envisioned combining formats of scavenger hunts and geocaching, adding narratives from popular sci-fi genres such as murder mystery, detective stories, and adventure games.

Some kind of virtual scavenger hunt aspect would be cool. There is still effort involved so it's not just a gimme and would likely be easy to incorporate team/group aspects as well as solo play. Maybe related to places you've been or just points within whatever game world. Kind of quest like, but with more difficult clues. - P1

Participants wanted to visit geographical locations virtually, partly because they felt limited in their ability to travel, but also because they were genuinely interested in visiting or learning more about different places. 
It could be cool for these ( \& any) players to be able to virtually play somewhere else. There are virtual field trips to places like Paris. How fun would it be to choose a virtual city to "visit" and use their 'Poke infrastructure'. - P6

A task related to visiting a cultural location [remotely] and get awarded something - Codesign Group 2 (P7, P9, P11)

Players were also interested in permanently keeping and showcasing game artifacts they found in locations that were meaningful to them. These included game objects they received from friends or keepsakes from their own travel that have interesting geographical origins or names. A few participants envisioned these keepsake artifacts as a learning experience.

Oh, I actually collect keys from travelers that come to my city or when I go abroad, like for I got keys from Antarctica and from Japan. And the one with the longest name, which I can't pronounce - a suggestion would be a feature that would store keepsakes separately if they do not plan [them] to be used. I wish we had a media locker because you know the artistic media from the First Saturday - they are so cute, but they take up so much space after a while. - P7

I know that one of my locals was talking for a long time about how much they would really like to have like a photo album or like a capsule where you could put special keys that you could never use again. - P9

\subsection{Reimagining Proximity-Based Social Connection}

As the pandemic made virtual communication necessary, players wanted in-game chats, remote trading, and in-game facilitation for coordinating with teams, knowing the presence of other players, and 'visiting' them virtually instead of using third party communication apps. Players emphasized creative ways to connect with people who are within safely distanced physical proximity while socially constructing a virtual "place" [8] for chatting and exchanging game items.

Social - an in-game chat for players within the distance of a [PoGO] Gym so people can coordinate raids, another chat for individual areas for general game discussion and trades Co-design Group 2 (P7, P9, P11)

PoGO: sending my buddy to go visit my friends nearby - Co-design Group 1 (P4, P5, P6)

Geocaching? Leave digital marks on portals so other players can do something with it - Codesign Group 2 (P7, P9, P11)

The ability to do item exchanges with other people without physically being near them. - P5

Players also envisioned role-playing in social interactions with distributed abilities and goals for different individuals to enhance the gameplay which ties back to creating and enhancing meaningful exploration and narratives in the physical and virtual place using AR. 
Table 2: Summary of themes in co-design sessions ( $\mathrm{S} 1$ is session 1, S2 is session 2, D is discord, highlighted ideas were elaborated by the participants)

\section{Exploration}

1. More immersive AR experience (e.g., [29]) (D)

2. Virtually play and visit locations remotely (D)

3. Keep souvenirs from locations or gifts without affecting inventory space (D)

4. Create locations that do not necessarily correspond to any physical location to allow resource gathering. (D)

5. Choose your own adventure AR type game (D)

6. History or location significance: historical portal hunting, visiting cultural location tasks, virtual museum exploration (S2)

7. Sharing location history - safety in location tracking in Ingress, indicating who has been there by leaving digital marks so others can do something with it, portals they have visited (S2)

\section{Social}

1. Ability to chat with others while waiting or playing on the knight bus (D)

2. Better facilitation to play with players you do not already know. For example, seeing when other game players are nearby who are not friends. (D)

3. Better visibility of other players' roles in $H P W U(\mathrm{D})$

4. Social or playing with friends (e.g., kudo system to thank each other and help support mental health, sending buddy to go visit friends nearby, location-based chat for people close to a gym and another for trades) (S2)

\section{Health}

1. Count physical activity other than walking toward in-game rewards (D)

2. Rewards for walking (e.g., Capsules for PoGO egg or gear spawns based on activity) (D)

3. People should have access from home to the closest gym and PokéStop without having to travel (D)

4. Adventure sync for other physical activities (e.g., be able to track distance traveled while in the same position but still active such as on a treadmill, improving on heart rate tracking/breathing tracking to log physical activities or relaxation) (D)

5. Physical exercise and distribution of screen time - indoors and rewards, Bluetooth enabled aids for games other than PoGO (S2)

6. More virtual events (including daily quests and at-home events since not everyone lives in a city) (S1)

7. Virtual movement (e.g., Knight bus) (S1)

8. Enhance home play options (e.g., virtual portal at home, more passcodes to help with recharging) (S1)

9. Adventure sync for home exercise progression (S1) 


\subsection{Expanding Healthy Physical \& Mental Health Practices}

Players envisioned gameplay through activities beyond requiring physical displacement to include at-home play. While they appreciated getting free remote rewards in the game during quarantine, they had a stronger desire for being able to "do something" in the game. In co-design session 1 , players brainstormed ideas on having a virtual "home portal" that can be used for obtaining resources without affecting the strategy of the game (e.g., cannot be attacked by the opposing team). Some wanted this to be like the Knight Bus in HPWU that could be accessed from any location, such as while waiting in line at the grocery store. The home portal, as explained by players, could also support mundane activities in the game for coping with stress.

Everyone has a virtual home portal. They can hack it but can't link to it. - Co-design Group $1(P 4, P 5, P 6)$

I think they're going to need to not be a limit on how often you can move it [virtual portal], or like the Knight Bus needs to not actually have a location. - P4

Some participants suggested alternative methods that allow for game progression through indoor physical activities in which the game can synchronize with the exercise equipment such as a treadmill. They wanted to include indoor physical activities and map their exercise metrics to virtual displacement in the game. Though with at-home workouts such as yoga and push-ups, participants were skeptical about the accuracy of devices that can track it or the possibility of cheating, as was also expressed in prior work [25].

One idea that comes to mind now is being able to move your location in Ingress any amount of distance that you've walked or jogged, even if it's on a treadmill. And once at the new location/portal, one should have the ability to take all actions otherwise allowable, just as if one was there in person. I believe many smartphones have the capability of detecting whether one is physically active vs. driving very slowly. Players get similar physical/social benefits while still adhering to any social distancing guidelines that may be in place. - P5

\section{DISCUSSION}

In this work, we focused on the empirical understanding of (1) the adjustments made by players of LBG and their communities during the COVID-19 pandemic as the key pillars of this genre (i.e., exploration, community, and health) are disrupted and (2) design needs envisioned by LBG players to improve support for exploration, community, and health. Here, we discuss design recommendations informed by these empirical needs and prior work and speculate how they might be implemented in future design of LBGs.

\subsection{Beyond Exploration: Supporting Social Construction of Places}

Harrison and Dourish discuss the concepts of "place" and "space", drawing from architectural and urban theories, to be applied to different domains in which collaborative work happens $[8,15]$. They describe space as "geometrical arrangements that might structure, constrain, and enable certain forms of movement and interaction" as opposed to place which are "settings [that] acquire recognizable and persistent social meaning in the course of interaction”. Ringland explains how 
the autistic Autcraft community constructed social meaning and tailored community interaction norms in the virtual "place" in Minecraft [50] and described this place as an integral part of the social reality of the player(s).

Many of the current LBGs are ubiquitous and aim to represent locations as "templated spaces" - the game can be played in different three-dimensional virtual environment overlaying the inreal-life topography in which gaming objects spawn and events occur to fit the location $[13,15,53]$. LBG activities occur simultaneously on the mobile device, in the physical space, and through meta-play such as information gathering and coordination on social media platforms [32]. In most LBG designs, however, there is an underlying assumption that we want to represent the "accurate" and "objective" facts about locations (e.g., concerns about the accuracy of the description of PokéStop). It is important to design LBGs as places that provide meaningful psychological experiences-called 'aura'-that players can associate with the physical space [34,53]. Most of the gaming activities are unevenly distributed in information-rich sites such as historic sites, actionrich spaces such as cities [63], or indoor spaces such as museums that may or may not be accessible. This creates challenges for engagement and construction of meaningful and enjoyable experiences due to personal differences (e.g., disability, safety concerns, or living in a rural area) or a global pandemic. The interpretation and aura of physical objects and spaces can be different for players depending on their background (e.g., whether they are local to a community, lived experiences, or beliefs).

There are other ways to represent locations in LBGs that are based more on people's interaction with the physical positions. For example, Chenoweth explains how "a city isn't just land, streets, and buildings, but places overlaid with the diverse history of many people" as she presents a topographic map of emotions representing feelings, stories and life experiences of people in Austin, Texas [21]. The XYZ Atlas was created by asking people to share locations in the city where they had their most significant emotional experiences, and the resulting map was able to document a variety of people's experience and stories with the locations [21].

Sequential storytelling can support the construction of meaningful socio-cultural experiences. Ingress has a complex narrative but is not always appealing or accessible to mainstream players. Pokémon and Harry Potter are established and larger story-driven franchises. During the pandemic, Niantic made attempts to add additional storytelling in PoGO and HPWU through antagonists. For example, Team Rocket brought antagonist challenges to PoGO players in hot air balloons [38]. Participants desired player-driven narratives in the local context.

Design Speculation: Local players can submit information that is not necessarily objective facts about the locations, but still meaningful to the individual and the community. This information should be tied to the existing physical artifacts [53]. For example, the game could let people know more about the person the memorial bench is named after. Similar to Missions ${ }^{5}$ in Ingress, let the players author and organize aspects of the game such as player-driven narratives in the local context, wherein player communities can put together a route and upload descriptions and images to guide other players, similar to tour guide experience.

${ }^{5}$ Missions in Ingress: https://fevgames.net/ingress/ingress-guide/ops/missions/ 
We see opportunities for LBGs where more subjective information about people's experiences with the locations can be preserved and shared among the community members. For example, many participants wished to keep mementos and demonstrated how they associate their experiences and emotions with places in real-life and digital objects in the game. Preserving, presenting, and sharing meaningful digital collectibles has become increasingly common among sports fans and gamers (e.g., [61,62]). In LBGs, these meaningful digital objects like keys or badges from different places allowed players to experience positive feelings from their own past interaction with the location or the person who gifted the digital object, even when they were not able to physically visit and explore these places.

Design Speculation: Players can collect digital representations of real locations and keep them separate to not negatively affect their or other's gameplay and inventory space [61]. Players can store, view or present, and share these digital memorabilia enabling them to recall good memories. If they are from friends, one can add a personal message with postcards to revisit shared positive memories experiences.

Note that participants stated how they like visiting real locations in different countries (even virtually) for the experience but also wanted a portal that allowed them to play but has no connection with an actual location other than its proximity to the player for efficiency in gameplay.

\subsection{Beyond Socializing: Leveraging Multiple Modalities of Social Gaming}

Socializing in augmented reality gaming distributes attention, roles, and modes of communication, which blends the boundaries of social and cultural norms in online and real world resulting in different forms engagement (and lack of engagement). Socializing in AR games can include active interactions (in-person or remote communication) or passive interactions (being physically copresent but not interacting in real-life watching others play). Schlieder et al. [51] discuss LBGs as opportunities for bringing in physical efforts of sports, and also talk about the need for solitary LBGs in certain situations (such as playing a game of geographically distributed tic-tac-toe across different city locations). The appeal of LBGs in scaffolding active social bridging and bonding experiences [4,56] was also a crucial driving factor for participants to continue gameplay and access support during difficult life circumstances. Behaviors of communities of practice [31] were evident as the communities quickly reacted to and put together plans to deal with various real life situations impacting the gameplay. For instance, players discussed how their feeling of closeness to other players was influenced by the frequent interactions with them in-real-life before the pandemic, often amplified by participating in offline events and gatherings.

During physical distancing, participants wanted to do additional things together at some common location virtually or while being safely distanced in-real-life, thus constructing social meaning and narratives within these physical spaces. Participants' ideas on asynchronous co-play prompt us to think more broadly about different affordances of social interactions, even considering the needs of those who are less social or cannot be in a physical proximity with others due to health issues who may prefer asynchronous and/or anonymous interactions. Examples include the antisocial hiking app: HOBBIT, where the app detected the presence of other hikers at 
a distance using WiFi scanners, warned the players, and supported hikers in planning their actions such as changing routes, taking breaks, or waiting for others to pass [47].

Design speculation: Players would benefit from knowing the presence of other players in proximity, in-game scaffolds for coordination, and increased range of interactions such as trading items or battling to allow them to safely distance and engage in social gaming activities in the virtual place.

Most of our participants shared how community members deeply care about each other and want additional ways to signal that in the game environment, not just for immediate friends but perhaps random players who are in the same neighborhood. This can enhance the sense of belonging in the community which was extremely important to our participants.

Design Speculation: Players can leave special items for other players in a particular digital location. These items cannot be collected by the player who is placing the items but can only be given to other players. Players can decide which friends get to see it and can set different time limits. To support remote interactions players could send their buddy Pokémon to checkin with a friend or team member. The friend can then respond and/or attach a gift and send the buddy back to the player.

These features can enhance reciprocity as also identified by Kim et al. [25] not just during physical distancing but also reduce the need for forced face-to-face interactions. These affordances would be beneficial for players who may be introverted or not want to actively engage [4] but might still benefit from not entirely withdrawing from social experiences.

\subsection{Beyond Physical Activity: Integrating Overall Health}

Participants' needs and ideas promote the notion of expanding support for health beyond physical movement to intentionally supporting lifestyle changes and mental wellbeing. The current design acted as catalysts for some players to integrate healthy behaviors in the players' daily lifestyle (at the "OS level") [58] as it distributed screen-time dependent gameplay with structural changes such as moving around, building social connections, and spending time outdoors. Designers need to be more intentional about a holistic view of health [25], reduce barriers by considering other kinds of activities that involve movement, use games to support and reinforce routine or regular growth, and improve mental wellbeing.

The participants in our study and Elis et al.'s large-scale survey study talked about using LBGs to gain a sense of normalcy and control and escape the stress of the pandemic as also found in other types of games $[9,17]$. The game features that support redundant and mundane interactions helped some participants relax and/or sleep. Synchronizing gameplay with real-world time in LBGs, such as stopping raids between certain time windows (already implemented in PoGO), also supports routine and motivation to get out of the house for players. Physical health goals in current LBGs are predetermined (e.g., walk $2.5 \mathrm{~km}, 5 \mathrm{~km}$, or $10 \mathrm{~km}$ to hatch eggs or unlock portmanteaus). Routine practices of wellbeing can also be supported by helping participants personalize their goals, by breaking down their goals to a manageable size incorporating the context of the participant and helping them work up to a bigger goal over time. This can also 
support casual players who may have quit due to not gaining satisfaction in accomplishing daily tasks or want to get back in the game but are too far behind other players.

Design Speculation: Players can be provided with multiple options to stay active and set their own goals and rituals. For example, the player may set their daily ritual as "walk $1 \mathrm{~km}$ every day to reach $10 \mathrm{~km}$ in 10 days" and the game can reinforce incremental growth by rewarding them.

A broader idea of health that encompasses mental wellbeing can include creative and feelgood elements or reminders to take care of oneself such as adequate sleep and food. For example, in Animal Crossing: New Horizons, non-playable characters check in on whether the player had lunch if they have been playing for long hours or encourage them to "stop running around and sit and enjoy the scenery for a while".

Design Speculation: A feature could be implemented to increase reciprocal interactions with the buddy. For example, after the player feeds the buddy, the buddy can in-turn catalyze selfcare practices by asking, "Thank you for feeding me, have you eaten today?" The game can also support affordances for indicating emotional states to selected friends (e.g., status of the buddy Pokémon indicates their mood or if they are taking a mental break) and allowing friends to non-intrusively show support when the player may be feeling emotionally upset. These can include options for adding personal messages to a gift in addition to stickers.

\subsection{Limitations and Future Work}

This study was conducted at the time when participants were adjusting to social distancing requirements during the COVID-19 pandemic, which influenced our data collection methods. We strived to attain diverse participation and make methods of participation accessible, however, only ten participants completed the co-design study. Future work should endeavor to focus on recruitment and increase the number of participants. These circumstances during the pandemic significantly focused the attention of participants on designing for quarantine and social distancing, which is reflected in the design ideas and sentiments gathered during the co-design sessions. In a post-pandemic world, other novel design ideas that move LBGs in new directions may become more salient. Lastly, the participants of this study skewed towards avid players of LBGs and thus, our findings reflect the perspective of those kinds of players as opposed to more casual players of LBGs. We derived these design implications from an in-depth user context of a small sample of location-based gamers who continued to use location-based games for their wellbeing during the pandemic. We see parallels in other research on LBG $[9,25,64]$ and the importance of place-making in mixed reality games [34,53]. This alignment with suggestions from existing studies indicates that the recommendations could perhaps benefit players more broadly, but further research will help better understand the applicability to broader player base and LBGs that are played on a smaller scale than Niantic games.

\section{CONCLUSION}

Participants envisioned the foundations of LBGs to help players get to know their communities, engage with each other, and become more physically active ended up becoming a means to 
exclude players due to many social and environmental factors and, amidst the COVID-19 pandemic, increased limitations, and deviations from normalcy. This study shows not only the challenges players encountered as they played these games in real-world contexts, but also opportunities that may not have been fully intended or expected by the developers (such as building job-related skills, helping people develop and express their identities, managing various mental health conditions) because of playing these games. By understanding lived experiences of players and re-envisioning the design of LBGs with players, we can better understand the games' impact and generate new ways for how they can become more inclusive and meaningful to players, even during challenging times. By engaging players who had grown to depend on the game to support their health and wellbeing to share and reflect on their experiences and generate new ideas for adjustments to features, we hope that this work can expand the potential benefits of these games. Our findings suggest that the supporting the social construction of space, designing for multiple modalities of engagement, and expanding to a broader notion of overall health are new ways that these games can achieve the goals initially articulated for these games. We hope that game designers can use these findings to expand their existing games and design new ones to provide a more inclusive, resilient, and varied LBG gaming experience.

\section{ACKNOWLEDGMENTS}

We are very grateful to all our participants who dedicated their time and ideas to this study despite their challenges during the COVID-19 Pandemic. We also thank anonymous reviewers for their feedback.

\section{REFERENCES}

[1] Paul JC Adachi and Teena Willoughby. 2017. The link between playing video games and positive youth outcomes. Child Development Perspectives 11, 3: 202-206.

[2] Nikolaos M Avouris and Nikoleta Yiannoutsou. 2012. A review of mobile location-based games for learning across physical and virtual spaces. J. UCS 18, 15: 2120-2142.

[3] Arpita Bhattacharya, Calvin Liang, Emily Y. Zeng, et al. 2019. Engaging Teenagers in Asynchronous Online Groups to Design for Stress Management. Proceedings of the 18th ACM International Conference on Interaction Design and Children, Association for Computing Machinery, 26-37.

[4] Arpita Bhattacharya, Travis W Windleharth, Rio Anthony Ishii, et al. 2019. Group interactions in location-based gaming: A case study of raiding in pokémon go. Proceedings of the 2019 CHI Conference on Human Factors in Computing Systems, 1-12.

[5] Max V Birk, Greg Wadley, Vero Vanden Abeele, Regan Mandryk, and John Torous. 2019. Video games for mental health. interactions 26, 4: 32-36.

[6] Ian Bogost. 2008. The rhetoric of video games. MacArthur Foundation Digital Media and Learning Initiative.

[7] Hafedh Chourabi, Taewoo Nam, Shawn Walker, et al. 2012. Understanding smart cities: An integrative framework. 2012 45th Hawaii international conference on system sciences, IEEE, 2289-2297.

[8] Paul Dourish. 2006. Re-space-ing place: " place” and" space" ten years on. Proceedings of the 200620 th anniversary conference on Computer supported cooperative work, 299-308.

[9] Louise A Ellis, Matthew D Lee, Kiran Ijaz, James Smith, Jeffrey Braithwaite, and Kathleen Yin. 2020. COVID-19 as 'Game Changer'for the Physical Activity and Mental Well-Being of Augmented Reality Game Players During the Pandemic: Mixed Methods Survey Study. Journal of medical Internet research 22, 12: e25117.

[10] James Paul Gee. 2006. Are video games good for learning? Nordic Journal of Digital Literacy 1, 03: 172-183.

[11] Meghan Gestos, Jennifer Smith-Merry, and Andrew Campbell. 2018. Representation of women in video games: a systematic review of literature in consideration of adult female wellbeing. Cyberpsychology, Behavior, and Social networking 21, 9: 535-541.

[12] Isabela Granic, Adam Lobel, and Rutger CME Engels. 2014. The benefits of playing video games. American psychologist 69, 1: 66 . 
[13] Anton Gustafsson, John Bichard, Liselott Brunnberg, Oskar Juhlin, and Marco Combetto. 2006. Believable environments: generating interactive storytelling in vast location-based pervasive games. Proceedings of the 2006 ACM SIGCHI international conference on Advances in computer entertainment technology, 24-es.

[14] Yemaya J Halbrook, Aisling T O’Donnell, and Rachel M Msetfi. 2019. When and how video games can be good: A review of the positive effects of video games on well-being. Perspectives on Psychological Science 14, 6: 10961104.

[15] Steve Harrison and Paul Dourish. 1996. Re-place-ing space: the roles of place and space in collaborative systems. Proceedings of the 1996 ACM conference on Computer supported cooperative work, 67-76.

[16] Kimihiro Hino, Yasushi Asami, and Jung Su Lee. 2019. Step counts of middle-aged and elderly adults for 10 months before and after the release of Pokémon GO in Yokohama, Japan. Journal of medical Internet research 21 2: e10724.

[17] Ioanna Iacovides and Elisa D Mekler. 2019. The Role of Gaming During Difficult Life Experiences. Proceedings of the 2019 CHI Conference on Human Factors in Computing Systems, 1-12.

[18] Ian Walker. 2021. Minecraft Earth Ends This Summer. Retrieved from https://kotaku.com/minecraft-earth-endsthis-summer-

1845992885?utm_campaign=Kotaku\&utm_content=1609870436\&utm_medium=SocialMarketing\&utm_source=fac ebook\&fbclid=IwAR1_CQJjMbCydkCps7gfgvHvDGuOLCxOcOqFFSokTHwelsArr7qHIVo3m28.

[19] Troy Innocent. 2018. Play about Place: Placemaking in location-based game design. Proceedings of the 4th Media Architecture Biennale Conference, 137-143.

[20] Jeroen Jansz and Martin Tanis. 2007. Appeal of playing online first person shooter games. Cyberpsychology \& behavior 10, 1: 133-136.

[21] Jennifer Chenoweth. 2017. Mapping the emotional topography of a community. Retrieved from https://www.tedmed.com/talks/show?id=688207.

[22] Jesse Emspak. 2018. What is Augmented Reality? Retrieved from https://www.livescience.com/34843-augmentedreality.html.

[23] Daniel Johnson, Sebastian Deterding, Kerri-Ann Kuhn, Aleksandra Staneva, Stoyan Stoyanov, and Leanne Hides. 2016. Gamification for health and wellbeing: A systematic review of the literature. Internet interventions 6: 89106.

[24] Lukas Dominik Kaczmarek, Michall Misiak, Maciej Behnke, Martyna Dziekan, and Przemyslaw Guzik. 2017. The Pikachu effect: Social and health gaming motivations lead to greater benefits of Pokémon GO use. Computers in Human Behavior 75: 356-363.

[25] Yoojung Kim, Arpita Bhattacharya, Julie A. Kientz, and Jin Ha Lee. 2020. "It Should Be a Game for Fun, Not Exercise": Tensions in Designing Health-Related Features for PokéMon GO. Proceedings of the $2020 \mathrm{CHI}$ Conference on Human Factors in Computing Systems, Association for Computing Machinery, 1-13.

[26] Samuli Laato, AKM Najmul Islam, and Teemu H Laine. 2020. Did location-based games motivate players to socialize during COVID-19? Telematics and Informatics 54: 101458.

[27] Samuli Laato, Teemu H Laine, and AKM Islam. 2020. Location-based games and the covid-19 pandemic: An analysis of responses from game developers and players. Multimodal Technologies and Interaction 4, 2: 29.

[28] Samuli Laato, Tarja Pietarinen, Sampsa Rauti, Mauri Paloheimo, Nobufumi Inaba, and Erkki Sutinen. 2019. A review of location-based games: Do they all support exercise, social interaction and cartographical training? CSEDU (1), 616-627.

[29] Lance Weiler. 2018. 13 Immersive Things - claymation games, AI theatre, VR/AR escape rooms, enchanted objects and more.... Retrieved from https://medium.com/columbia-dsl/13-immersive-things-claymation-games-aitheatre-vr-ar-escape-rooms-enchanted-objects-and-more-d05c15b06ca.

[30] Grace Lau and Phil Agre. 2005. Developing online communities of practice: A case study of the World of Warcraft. Information Policy Seminar, Citeseer.

[31] Jean Lave, Etienne Wenger, and others. 1991. Situated learning: Legitimate peripheral participation. Cambridge university press.

[32] Jin Ha Lee, Stephen A Keating, and Travis W Windleharth. 2017. Challenges in Preserving Augmented Reality Games: A Case Study of Ingress and Pokémon GO. iPRES.

[33] Adam Lobel, Rutger CME Engels, Lisanne L Stone, William J Burk, and Isabela Granic. 2017. Video gaming and children's psychosocial wellbeing: A longitudinal study. Journal of youth and adolescence 46, 4: 884-897.

[34] Blair MacIntyre, Jay David Bolter, and Maribeth Gandy. 2004. Presence and the aura of meaningful places. Presence: Teleoperators and Virtual Environments 6, 2: 197-206.

[35] Haley MacLeod, Ben Jelen, Annu Prabhakar, Lora Oehlberg, Katie Siek, and Kay Connelly. 2017. A Guide to Using Asynchronous Remote Communities (ARC) for Researching Distributed Populations. EAI Endorsed Transactions on Pervasive Health and Technology 17, 11.

[36] MANSOOR IQBAL. 2021. Pokémon Go Revenue and Usage Statistics (2021). Retrieved from https://www.businessofapps.com/data/pokemon-go-statistics/.

[37] Megan Mathers, Louise Canterford, Tim Olds, Kylie Hesketh, Kate Ridley, and Melissa Wake. 2009. Electronic media use and adolescent health and well-being: cross-sectional community study. Academic pediatrics 9, 5: 307314. 
[38] Megan Farokhmanesh. 2020. POKÉMON GO CREATORS SAY COVID IS 'EXISTENTIAL TO OUR GAME': A pandemic makes pushing your players outside impossible. Retrieved from https://www.theverge.com/2020/12/19/22189424/pokemon-go-covid-2020-safety.

[39] Pauline H Mosley and Jayfus T Doswell. 2008. The Virtual Instructor Intervention: A Case in LEGO Robotics. IJVR 7, 1: 15-20.

[40] Niantic. Our Technology Brings People Together. Retrieved from https://nianticlabs.com/en/social-impact/.

[41] Daniela Nicklas, Matthias Großmann, Thomas Schwarz, Steffen Volz, and Bernhard Mitschang. 2001. A modelbased, open architecture for mobile, spatially aware applications. International Symposium on Spatial and Temporal Databases, Springer, 117-135.

[42] Rune KL Nielsen and Daniel Kardefelt-Winther. 2018. Helping parents make sense of video game addiction. In Video Game Influences on Aggression, Cognition, and Attention. Springer, 59-69.

[43] Anton Nijholt. 2017. Towards playful and playable cities. In Playable Cities. Springer, 1-20.

[44] Diana Oblinger and Joan K Lippincott. 2006. Learning spaces. Boulder, Colo,.: EDUCAUSE, c2006. 1 v.(various pagings): illustrations.

[45] Federica Pallavicini, Ambra Ferrari, and Fabrizia Mantovani. 2018. Video games for well-being: A systematic review on the application of computer games for cognitive and emotional training in the adult population. Frontiers in psychology 9: 2127.

[46] Anthony Pellicone and June Ahn. 2014. Construction and community: Investigating interaction in a Minecraft affinity space. Proceedings of the Tenth Conference for Games+ Learning+ Society, 187-193.

[47] Maaret Posti, Johannes Schöning, and Jonna Häkkilä. 2014. Unexpected journeys with the HOBBIT: the design and evaluation of an asocial hiking app. Proceedings of the 2014 conference on Designing interactive systems, 637-646.

[48] Ruth Potts and Lachlan Yee. 2019. Pokémon Go-ing or staying: exploring the effect of age and gender on augmented reality game player experiences in public spaces. Journal of Urban Design 24, 6: 878-895.

[49] Andrew K Przybylski, Netta Weinstein, Richard M Ryan, and C Scott Rigby. 2009. Having to versus wanting to play: Background and consequences of harmonious versus obsessive engagement in video games. CyberPsychology \& Behavior 12, 5: 485-492.

[50] Kathryn E. Ringland. 2019. A Place to Play: The (Dis)Abled Embodied Experience for Autistic Children in Online Spaces. Proceedings of the 2019 CHI Conference on Human Factors in Computing Systems, Association for Computing Machinery, 1-14.

[51] Christoph Schlieder, Peter Kiefer, and Sebastian Matyas. 2006. Geogames: Designing location-based games from classic board games. IEEE Intelligent Systems 21, 5: 40-46.

[52] Ben Schouten, Gabriele Ferri, Michiel de Lange, and Karel Millenaar. 2017. Games as strong concepts for citymaking. In Playable Cities. Springer, 23-45.

[53] Hitesh Nidhi Sharma, Sultan A Alharthi, Igor Dolgov, and Zachary O Toups. 2017. A framework supporting selecting space to make place in spatial mixed reality play. Proceedings of the Annual Symposium on ComputerHuman Interaction in Play, 83-100.

[54] John L Sherry, Kristen Lucas, Bradley S Greenberg, and Ken Lachlan. 2006. Video game uses and gratifications as predictors of use and game preference. Playing video games: Motives, responses, and consequences 24, 1: 213-224.

[55] Christos Sintoris, Nikoleta Yiannoutsou, and Nikolaos Avouris. 2017. Design of Location-Based Mobile Games: Introduction. IFIP Conference on Human-Computer Interaction, Springer, 379-382.

[56] Kiley Sobel, Arpita Bhattacharya, Alexis Hiniker, Jin Ha Lee, Julie A Kientz, and Jason C Yip. 2017. It wasn't really about the PokéMon: parents' perspectives on a location-based mobile game. Proceedings of the $2017 \mathrm{CHI}$ Conference on Human Factors in Computing Systems, 1483-1496.

[57] Statista Research Department. 2016. Number of daily Pokémon GO users in the United States as of August 2016. Retrieved from https:/www.statista.com/statistics/589213/pokemon-go-user-numberus/\#: :text=The\%20graph\%20shows\%20the\%20number,GO\%20had\%2030\%20million\%20DAU.

[58] Steve Downs. 2017. How technology can shape our lives - for the better. Retrieved from https://tincture.io/itstime-to-build-health-into-the-os-1783e118b54d.

[59] Laura Stockdale and Sarah M Coyne. 2018. Video game addiction in emerging adulthood: Cross-sectional evidence of pathology in video game addicts as compared to matched healthy controls. Journal of affective disorders 225: 265-272.

[60] Tamara D Street, Sarah J Lacey, and Rebecca R Langdon. 2017. Gaming your way to health: A systematic review of exergaming programs to increase health and exercise behaviors in adults. Games for Health Journal 6, 3: 136146.

[61] Zachary O Toups, Nicole K Crenshaw, Rina R Wehbe, Gustavo F Tondello, and Lennart E Nacke. 2016. “ The Collecting Itself Feels Good" Towards Collection Interfaces for Digital Game Objects. Proceedings of the 2016 Annual Symposium on Computer-Human Interaction in Play, 276-290.

[62] Diane Watson, Deltcho Valtchanov, Mark Hancock, and Regan Mandryk. 2014. Designing a gameful system to support the collection, curation, exploration, and sharing of sports memorabilia. Proceedings of the first ACM SIGCHI annual symposium on Computer-human interaction in play, 451-452. 
[63] Richard Wetzel, Lisa Blum, and Leif Oppermann. 2012. Tidy city: a location-based game supported by in-situ and web-based authoring tools to enable user-created content. Proceedings of the international conference on the foundations of digital games, 238-241.

[64] Travis W Windleharth. 2020. Identity, Safety, and Information Management within Communities of Practice in Location-based Augmented Reality Games: A Case Study of Ingress. .

[65] Annika Wolff, Gerd Kortuem, and Jose Cavero. 2015. Urban data games: Creating smart citizens for smart cities. 2015 IEEE 15th International Conference on Advanced Learning Technologies, IEEE, 164-165.

[66] Nan Zeng and Zan Gao. 2016. Exergaming and obesity in youth: current perspectives. International journal of general medicine 9: 275 .

Received February 2021; revised June 2021; accepted July 2021. 